\title{
Pour bien commencer les communications numériques : écoutez la radio en qualité numérique !
}

\author{
Hervé BOEGLEN $^{1}$, Laurent MURA ${ }^{2}$, Christian CHATELLIER $^{3}$, Olivier HAEBERLE ${ }^{1}$ \\ ${ }^{l}$ Laboratoroire MIPS LABEL, IUT Mulhouse, 61 rue Albert Camus, 68093 Mulhouse Cedex, France \\ ${ }^{2} I U T$ de Colmar, Département R\&T, 34, rue du Grillenbreit, 68008 Colmar Cedex, France \\ ${ }^{3}$ Laboratoire SIC XLIM, UMR CNRS 6172, Téléport 2, Bd M. et P. Curie, 86962 Futuroscope Cedex, France
}

herve.boeglenluha.fr

\begin{abstract}
Résumé : Dans cet article, nous présentons un système de radio numérique basé sur la norme Digital Radio Mondiale (DRM). Ce système comprend une partie logicielle dont le code source est libre et une partie matérielle d'un coût très réduit (moins de 60€). Il est utilisé pour illustrer de manière concrète les enseignements de communications numériques et d'électronique des transmissions dispensés en $2^{\text {2èe }}$ année de DUT Réseaux et Télécommunications $(R \& T)$.
\end{abstract}

Mots clés : Transmissions numériques, électronique des transmissions, télécommunications

\section{Introduction :}

Depuis environ cinq ans, nous sommes confrontés à un nombre grandissant d'étudiants imperméables aux notions théoriques qui s'appuient sur des exposés mathématiques. Il s'en suit un manque de motivation pour les matières que nous enseignons, en l'occurrence l'électronique et les transmissions numériques.

Pour faire face à ce problème de motivation, nous avons entrepris une démarche pédagogique basée sur l'étude et la mise en œuvre de systèmes réels. Grâce à cette approche pratique, nous parvenons à motiver les étudiants d'une part, et à les convaincre que la conception et la réalisation d'un système réel nécessite de bonnes connaissances théoriques dans les différentes disciplines liées aux télécommunications d'autre part. Le système de radio numérique que nous présentons dans cet article est un exemple d'application de cette démarche qui connaît un réel succès auprès des étudiants de $2^{\text {ème }}$ année de DUT Réseaux et Télécommunications (R\&T) de l'IUT de Colmar.

La partie 2 de cet article décrit la norme de radio numérique DRM. Dans la partie 3 nous décrivons la mise en œuvre d'un émetteur-récepteur DRM à $4,3 \mathrm{MHz}$. Enfin dans la dernière partie nous décrivons l'exploitation du système DRM dans le cadre des enseignements d'électronique et de transmission numérique de $2^{\text {ème }}$ année de DUT R\&T.
2. La norme de radio numérique Digital Radio Mondiale (DRM) :

Le système DRM [1] a été conçu pour remplacer les systèmes de radiodiffusion analogiques AM dans la bande des $300 \mathrm{kHz}-$ $30 \mathrm{MHz}$. DRM apporte des améliorations significatives en terme de qualité audio et de robustesse de transmission. Il s'agit d'un système de communication numérique à bas débit qui permet des émissions audio en qualité stéréo (compression audio MPEG4) tout en permettant la transmission d'informations multimédia (images, news, bulletins météo etc.).

Pour faire face aux conditions de propagation particulières à ces gammes de fréquence, la norme fixe différents modes de transmission qui peuvent être classés selon deux critères :

- la bande passante

- la robustesse de la transmission

Le premier critère définit la largeur de bande de transmission. En ce qui concerne le deuxième critère, on se préoccupe du compromis entre la capacité (débit utile) et la résistance au bruit, aux multitrajets et à l'effet Doppler. En effet, la norme prend en compte la mobilité possible du récepteur (en voiture par exemple). 


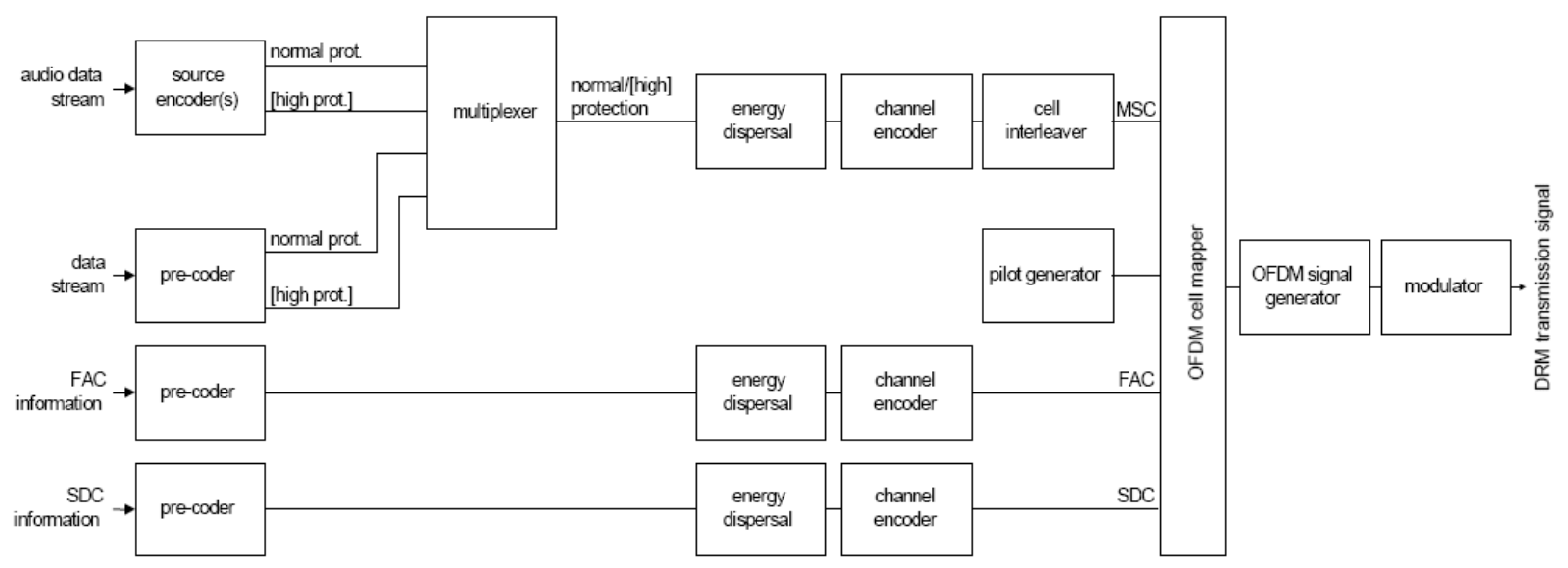

Figure 1 : Bloc diagramme conceptuel d'une transmission DRM

\subsection{Architecture du système:}

La transmission DRM comporte deux niveaux de codage / décodage successifs (cf. Figure 1) :

- Un codage logique (audio, data) qui constitue le Main Service Channel (MSC). On peut transmettre jusqu'à quatre canaux simultanés comprenant de l'audio codé en MPEG4-AAC et des données multimédia (images, news etc.). Le Service Description Channel (SDC) contient les informations relatives aux services disponibles sur le MSC avec des informations détaillées sur comment le récepteur doit décoder chacun des services (audio, audio-stéréo, multilingue, multimédia, site web, etc.)

- Un codage physique qui permet l'adaptation au canal de transmission. Il constitue le Fast Access Channel (FAC) qui contient les informations pour piloter le récepteur (largeur de bande, nature de la modulation, mode de protection, etc.). Il est spécifié sur la largeur de bande minimale, définie dans le standard DRM, en l'occurrence 4,5 kHz. Le signal transmis est structuré à la fois en fréquence et en temps (cf. Fig. 2).
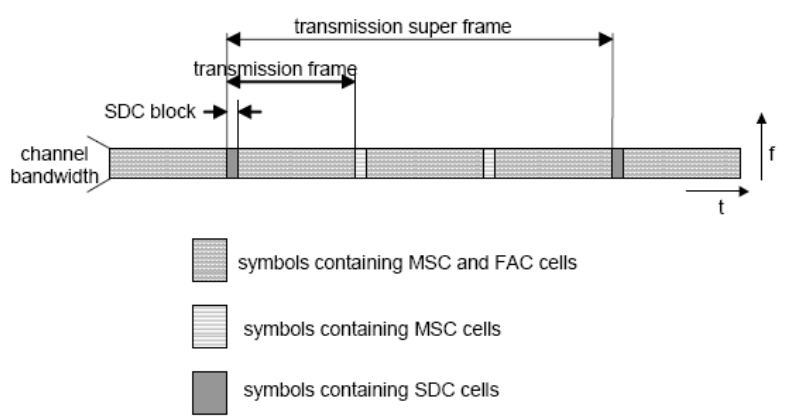

Figure 2: Positionnement temps-fréquence des signaux FAC et $S D C$ du signal DRM

Le standard stipule quatre modes de robustesse qui dépendent des conditions de propagation (cf. Fig. 3).

\begin{tabular}{|c|l|}
\hline Robustness mode & \multicolumn{1}{|c|}{ Typical propagation conditions } \\
\hline A & Gaussian channels, with minor fading \\
\hline B & Time and frequency selective channels, with longer delay spread \\
\hline C & As robustness mode B, but with higher Doppler spread \\
\hline D & As robustness mode B, but with severe delay and Doppler spread \\
\hline
\end{tabular}

\section{Figure 3 : Modes de robustesse DRM}

\subsection{Structure du signal DRM :}

Pour faire face à la sélectivité fréquentielle des canaux de transmission ionosphériques utilisés, DRM utilise l'OFDM [2].

Dans le domaine temporel, le signal est constitué d'une «supertrame» de durée $1,2 \mathrm{~s}$, elle-même contenant trois trames de durée $400 \mathrm{~ms}$ (groupe de 15 à 24 symboles OFDM). Entre chaque supertrame est intercalé un bloc SDC. Chaque symbole de durée Ts a une durée utile Tu et un intervalle de garde de durée Tg (cf. Fig. 4).

\begin{tabular}{|l|c|c|c|c|}
\hline \multirow{2}{*}{ Parameters list } & \multicolumn{4}{|c|}{ Robustness mode } \\
\cline { 2 - 5 } & A & B & C & D \\
\hline$T(\mu \mathrm{s})$ & $83^{1 / 3}$ & $83^{1 / 3}$ & $83^{1 / 3}$ & $83^{1 / 3}$ \\
\hline$T_{u}(\mathrm{~ms})$ & 24 & $21^{1 / 3}$ & $14^{2 / 3}$ & $9^{1 / 3}$ \\
\hline$T_{g}(\mathrm{~ms})$ & $(288 \times T)$ & $(256 \times T)$ & $(176 \times T)$ & $(112 \times T)$ \\
\hline$T_{g} / T_{u}$ & $2^{2 / 3}$ & $5^{1 / 3}$ & $5^{1 / 3}$ & $7^{1 / 3}$ \\
\hline$T_{s}=T_{u}+T_{g}(\mathrm{~ms})$ & $12 \times T)$ & $(64 \times T)$ & $(64 \times T)$ & $(88 \times T)$ \\
\hline$T_{f}(\mathrm{~ms})$ & $26^{2 / 3}$ & $1 / 9$ & $4 / 11$ & $11 / 14$ \\
\hline
\end{tabular}

Figure 4 : Paramètres des symboles OFDM

Dans le domaine fréquentiel, chaque symbole OFDM est constitué de $\mathrm{K}$ porteuses (cf. Fig. 5). Chaque porteuse est modulée en M-QAM avec M pouvant varier de 4 à 64 .

\begin{tabular}{|c|c|c|c|c|c|c|c|}
\hline \multirow{2}{*}{$\begin{array}{l}\text { Robustness } \\
\text { mode }\end{array}$} & \multirow[t]{2}{*}{ Carrier } & \multicolumn{6}{|c|}{ Spectrum occupancy } \\
\hline & & 0 & 1 & 2 & 3 & 4 & 5 \\
\hline \multirow[t]{2}{*}{ A } & $\mathrm{K}_{\min }$ & 2 & 2 & -102 & -114 & -98 & -110 \\
\hline & $\mathrm{K}_{\max }$ & 102 & 114 & 102 & 114 & 314 & 350 \\
\hline \multirow[t]{2}{*}{ B } & $\mathrm{K}_{\min }$ & 1 & 1 & -91 & -103 & -87 & -99 \\
\hline & $\mathrm{K}_{\max }$ & 91 & 103 & 91 & 103 & 279 & 311 \\
\hline \multirow[t]{2}{*}{ c } & $\mathrm{K}_{\min }$ & - & - & - & -69 & - & -67 \\
\hline & $\mathrm{K}_{\max }$ & - & - & - & 69 & - & 213 \\
\hline \multirow[t]{2}{*}{ D } & $\mathrm{K}_{\min }$ & - & - & - & -44 & - & -43 \\
\hline & $\mathrm{K}_{\max }$ & - & - & - & 44 & - & 135 \\
\hline
\end{tabular}

Figure 5 : Nombre de porteuses pour chaque mode 


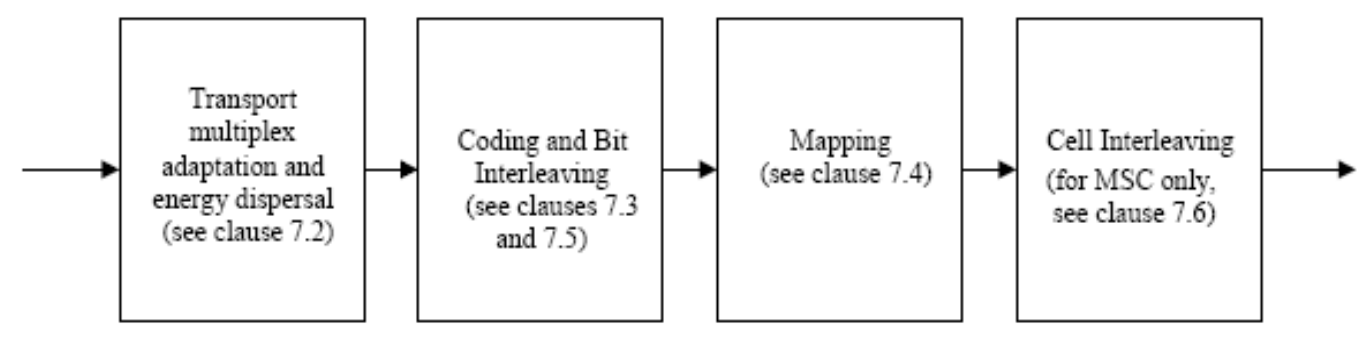

Figure 6 : Bloc diagramme du codage et de l'entrelacement

\subsection{Codage Correcteur d'Erreurs (CCE) et paramètres d'estimation du canal :}

Le CCE est basé sur l'utilisation d'un code convolutif appelé code mère dont le rendement varie grâce à l'utilisation d'une matrice de poinçonnage. Plus le mode de transmission est robuste et plus le rendement est faible. Ce CCE est associé à un procédé de mapping des constellations QAM appelé codage multiniveaux (multilevel coding) :

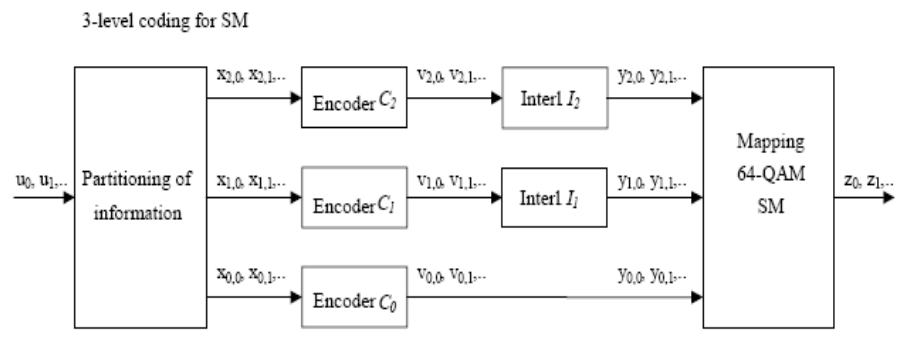

Figure 7 : Multilevel coding pour le mapping standard

Pour éviter les erreurs en rafales, fréquentes sur les canaux à évanouissements, les données entrent dans un entrelaceur matriciel dont la taille et le schéma de permutation dépendent du mode de robustesse choisi.

Enfin, pour permettre au récepteur d'estimer le canal de transmission, des symboles pilotes sont insérés dans la direction fréquentielle à des positions déterminées (cf. Fig. $8)$.

\begin{tabular}{|c|c|}
\hline Robustness mode & Carrier numbers \\
\hline A & $18,54,72$ \\
\hline B & $16,48,64$ \\
\hline C & $11,33,44$ \\
\hline D & $7,21,28$ \\
\hline
\end{tabular}

Figure 8 : Position des pilotes dans le symbole OFDM

\section{Description de la manipulation :}

Elle est constituée d'un émetteur et d'un récepteur. Dans les deux cas, on dispose d'une partie logicielle et d'une partie matérielle.

\subsection{Emission :}

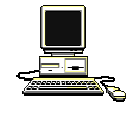

$\mathrm{PC}$

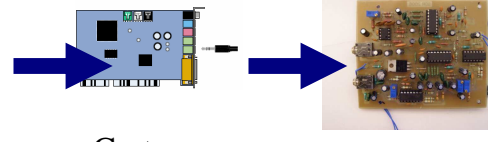

Carte son PC
Module HF

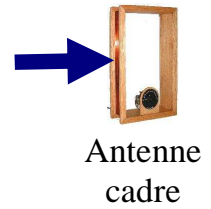

Figure 9 : Schéma de principe de l'émetteur
L'émetteur est constitué d'un PC et d'un module HF assurant la modulation d'amplitude en Bande Latérale Unique (BLU) et la translation de spectre autour de $4,3 \mathrm{MHz}$. Les faibles débits mis en œuvre (de l'ordre de 20kbits/s) permettent l'implémentation logicielle du système en bande de base. Cette partie logicielle appelée DREAM a été développée par deux chercheurs de l'Université de Darmstadt et est disponible sous licence GPL [3]. En lançant le logiciel avec l'option -t, on passe en mode émission (cf. Fig. 10) et l'on peut sélectionner le type de transmission (son ou image) ainsi que les différents paramètres du codage de canal. Le signal temps réel modulé en OFDM est alors disponible à la sortie de la carte son du PC émetteur.

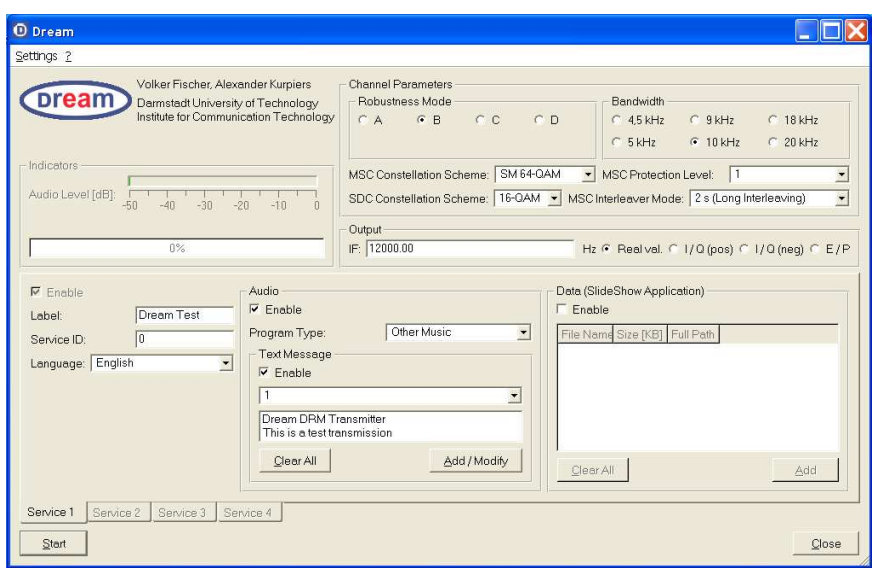

Figure 10: Logiciel DREAM en émission DRM

Pour pouvoir s'affranchir de l'absence d'une émission «live» [4], nous avons développé un module émetteur de faible puissance $(+20 \mathrm{dBm})$ conforme au schéma blocs de la Fig. 11.

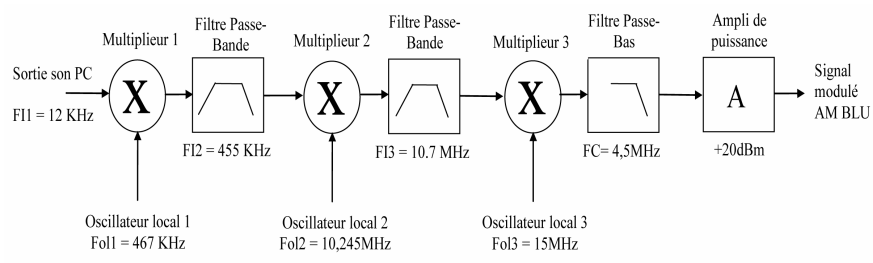

Figure 11 : Schéma blocs de l'émetteur DRM BLU

L'émission DRM doit être de type modulation d'amplitude (AM) en Bande Latérale Unique (BLU) mais on peut se contenter d'une émission AM classique (qui peut être obtenue à l'aide d'un simple générateur de fonction) en prenant soin de fixer la fréquence de démodulation sur l'une des bandes latérales (cf. Fig. 12). On remarquera que dans le cas de la Fig. 12 la largeur du spectre DRM est de 10kHz. 


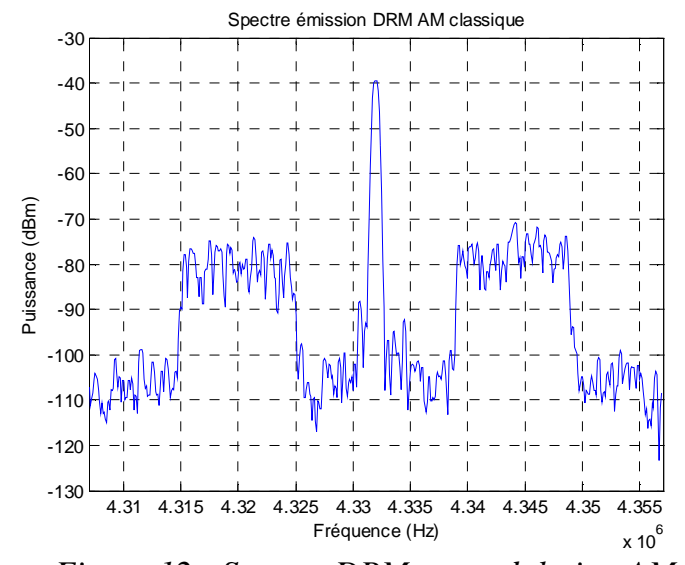

Figure 12 : Spectre DRM en modulation AM

La sortie de l'émetteur est reliée à une antenne cadre accordée par condensateur variable sur 4,3MHz (cf. Fig. 13). Cette partie a été conçue pour des puissances d'émission pouvant atteindre $10 \mathrm{~W}$ (puissance non utilisée car non autorisée !).

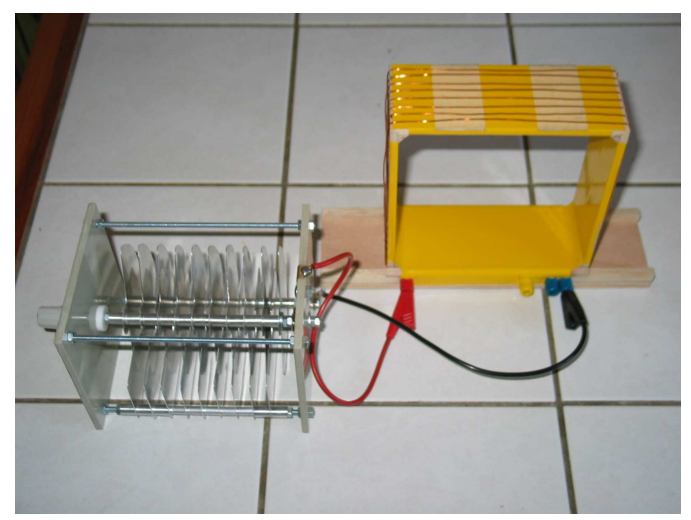

Figure 13 : Antenne d'émission et condensateur d'accord

\subsection{Réception :}

Le récepteur est précédé d'une antenne à cadre de ferrite accordable complétée par un étage d'adaptation à transistor à effet de champ (cf. Fig. 14).

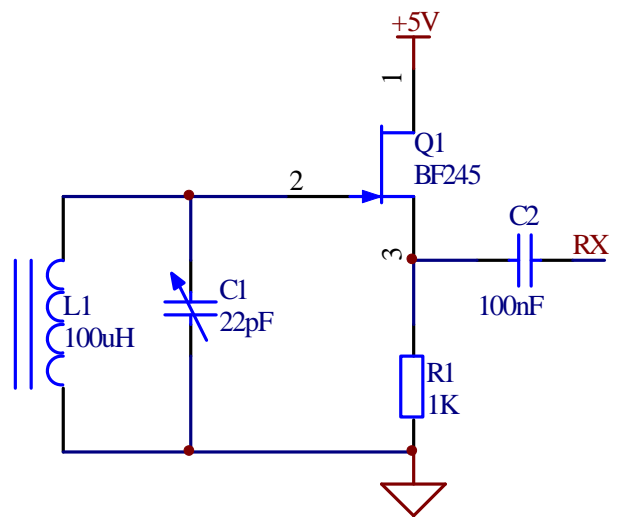

Figure 14 : Schéma de la tête HF de réception

Le récepteur qui assure la translation de spectre de $1-20 \mathrm{MHz}$ vers une fréquence intermédiaire (FI) de $12 \mathrm{KHz}$ est issu de la revue Elektor [5] et est conforme au schéma blocs de la Fig. 16.

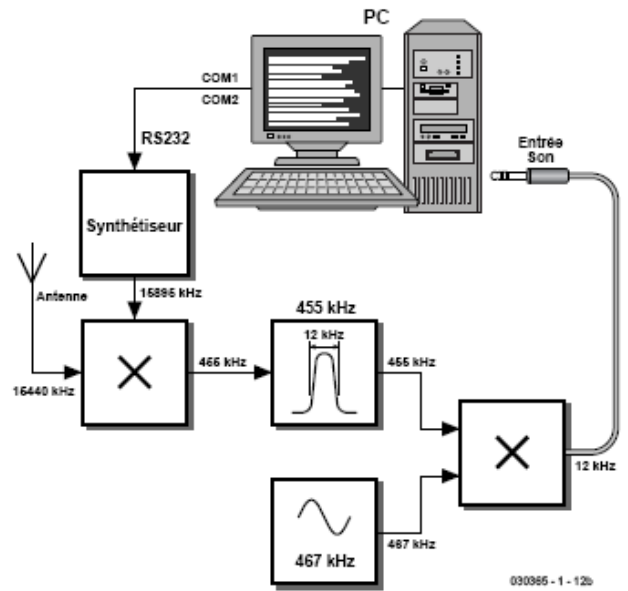

Figure 15 : Schéma blocs du récepteur DRM Elektor

Les fonctions de démodulation et de décodage sont assurées par le logiciel DREAM fonctionnant en mode réception. Il permet d'accéder aux différents paramètres de la réception OFDM : algorithmes d'estimation de canal, affichage des constellations etc., tout en écoutant la radio en qualité numérique !

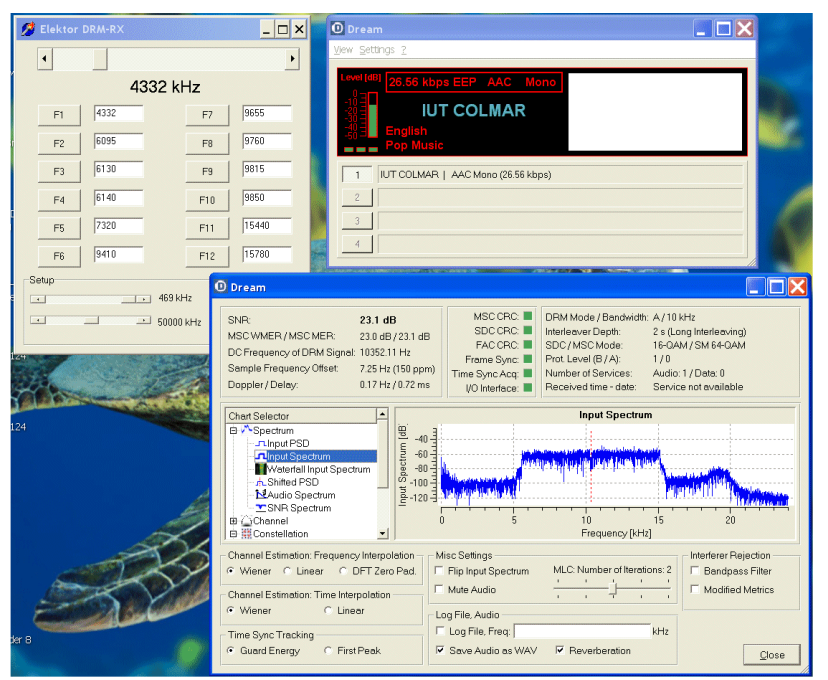

Figure 16: Logiciel DREAM en réception audio

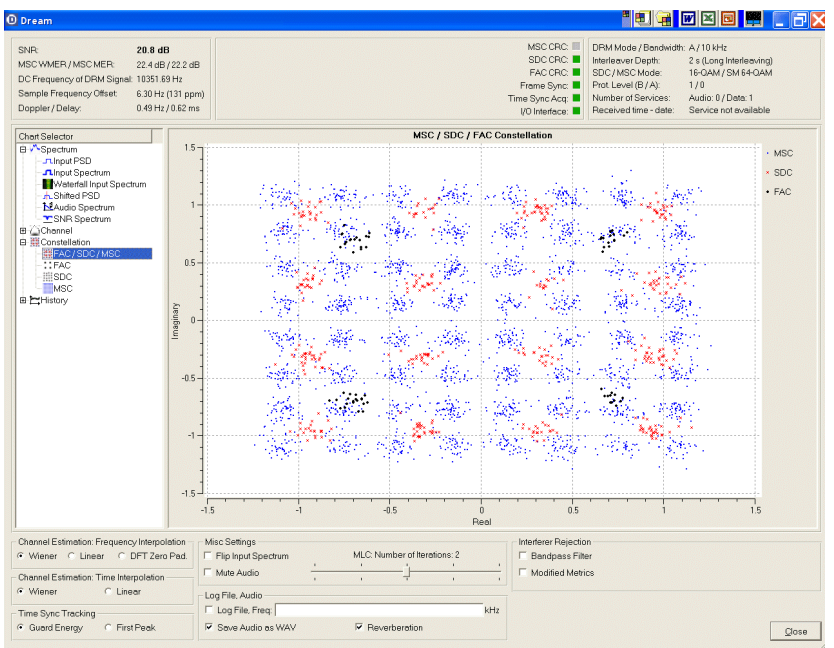

Figure 17 : Logiciel DREAM : affichage des constellations MSC (64QAM), SDC (16QAM) et FAC (4QAM) 


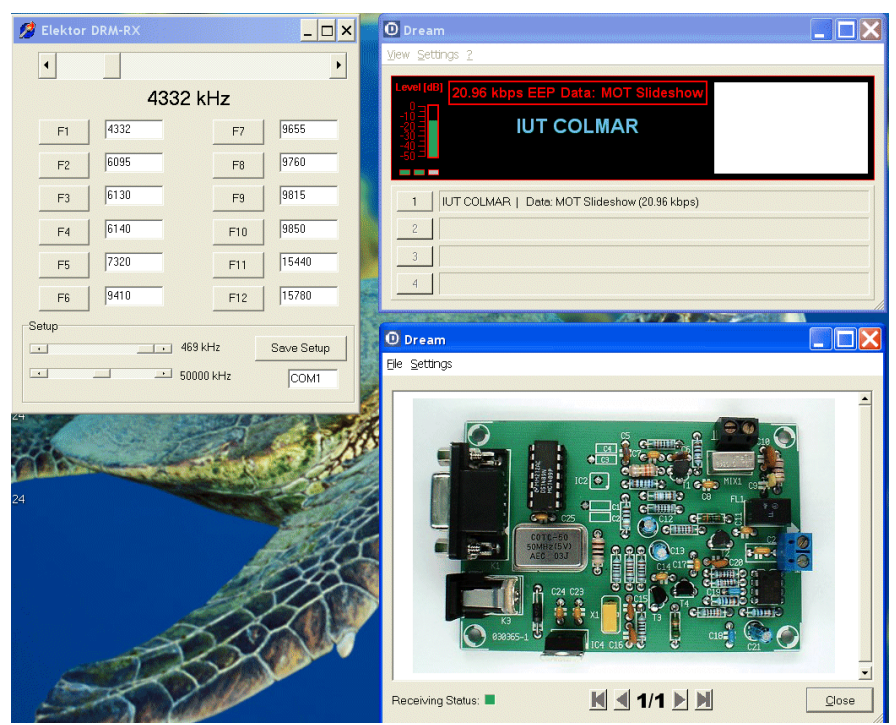

Figure 18 : Logiciel DREAM : réception de l'image JPEG du récepteur DRM Elektor

\section{Exploitation pédagogique en $2^{\text {ème }}$ année de DUT R\&T:}

L'intérêt pédagogique du système émetteur-récepteur DRM est important puisqu'il fait appel à nombre de notions et de compétences traitées dans le programme pédagogique R\&T. En particulier :

- $\quad$ Electronique [E4 et EC4] :

○ PLL

- Structure hétérodyne

- Antennes

○ Ampli HF

- Transmission numérique [TR1] :

- Modulations numériques

○ CCE

- OFDM

- Canal radio

- Estimation de canal

Le système est utilisé de deux manières différentes. La première dans un cadre de démonstration d'une notion particulière lors d'un cours magistral ou d'une séquence de travaux dirigés. Dans le cas du cours d'électronique, lors de la présentation de la notion de structure hétérodyne, par exemple, l'enseignant montre grâce à un analyseur de spectre les différentes translations fréquentielles que subit le signal DRM de l'émission à la réception. Dans le cours de transmission numérique, la difficile notion de sélectivité fréquentielle que subit le signal DRM ainsi que le moyen de la combattre à l'aide de l'OFDM sont présentées de manière très concrète grâce à une démonstration du système DRM. Des exemples de simulations dynamiques sont donnés avec Simulink [6] pour bien visualiser les aspects essentiels.

La deuxième exploitation se fait dans le cadre de deux séances de Travaux Pratiques (TP) intégrés dans une séquence de 6 TP tournants de transmission numérique.

Le premier TP se focalise sur les aspects liés à la couche physique radiofréquence. Ce TP s'articule autour de 3 notions importantes :
- Le changement de fréquence dans une structure hétérodyne. Cette notion est illustrée par l'étude du module HF d'émission (cf. Figure 11) en pratiquant les mesures adéquates.

- L'influence des antennes sur la qualité de la transmission. En ce qui concerne l'émission, les étudiants justifient l'utilisation d'une antenne cadre à la fréquence de $4,3 \mathrm{MHz}$ et vérifient la notion d'accord à partir du condensateur variable (cf. Figure 13). Au niveau de la réception, ils mesurent le niveau du signal sur l'étage d'entrée et à partir du logiciel DREAM en mode réception valident l'importance de la notion de Rapport Signal sur Bruit.

- L'encombrement spectral. A partir du logiciel DREAM en mode émission, du module HF étudié précédemment et d'un analyseur de spectre, l'étudiant étudie le spectre du signal DRM selon les différents modes prévus dans le standard. L'objectif est d'observer la forme d'un spectre OFDM et de comprendre la relation entre bande occupée et débit binaire.

Le deuxième TP, quant à lui, consiste d'une part en la mise en œuvre complète d'un émetteur-récepteur DRM à l'aide des modules décrits dans les parties précédentes et d'autre part en l'étude des performances du système pour différentes conditions de transmission. Le logiciel DREAM en mode émission permet de simuler différentes conditions de transmission. En effet, il implémente en temps réel les différents modèles de canaux décrits dans le standard: du canal Gaussien jusqu'à des modèles sélectifs en temps (effet Doppler) et en fréquence. A partir de ces conditions et du choix des modes de robustesse adéquats, l'étudiant est en mesure :

- D'évaluer les performances des différentes modulations numériques implémentées dans le standard.

- De comprendre l'influence des différents codes correcteurs d'erreurs implémentés et notamment de lier la notion de rendement de codage à celle de la robustesse du code.

Ces notions sont d'autant mieux intégrées que le système est entièrement fonctionnel: le mauvais choix d'un mode de robustesse se traduira par des perturbations ou une interruption de la diffusion.

Enfin, les étudiants évaluent les possibilités multimédia du système DRM en transmettant des images JPEG d'une taille d'environ 50k/octets. Cela leur permet de prendre conscience que le fait de considérer des données binaires permet de transmettre facilement différents types de supports numérisés (son, images, news etc.). D'autre part cette partie permet de se rendre compte que DRM est un système bas débit et que le temps de transmission d'une image dépasse la seconde. Cet élément permet d'introduire la notion de compression et de prendre conscience de son importance. 
Finalement, la diversité de ce système, dans les aspects qu'il met en œuvre, montre bien que malgré le découpage des modules en disciplines distinctes, il y a forcément transversalité d'un module à un autre, et d'une matière à une autre. Cette caractéristique pédagogique est un atout supplémentaire qui contribue au développement de la motivation de nos étudiants.

\section{Conclusion :}

Nous avons présenté un système de transmission numérique qui permet d'illustrer de nombreux concepts présentés dans le programme du DUT R\&T de manière ludique puisque l'on dispose d'un système de radio numérique parfaitement fonctionnel. De plus ce système présente l'avantage d'un faible coût (moins de $60 €$ pour les modules HF d'émission et de réception), puisqu'il est en général facile de disposer de deux ordinateurs de type PC basiques.

\section{Références :}

[1] ETSI ES 201980 V2.2.1 DRM: System Specification

[2] J. Bingham, Multicarrier modulation for transmission: an idea whose time has come, IEEE communications magazine, Volume 28, Issue 5, pp. 5-14, May 1990

[3] http://drm.sourceforge.net/

[4] http://www.drm.org/

[5] Récepteur DRM maison : Elektor n³ 309, mars 2004.

[6] http://www.mathworks.com 